\title{
Einführung TARMED im UVG
}

Dr. med. H. H. Brunner, Präsident FMH

Bezüglich der Einführung des neuen UV/MV/IVTarifs (UMIT) informieren wir Sie wie folgt:

1. Die Abrechnungsformulare für alle eidgenössischen Sozialversicherer sind bereinigt und in Produktion gegangen; sie werden fristgerecht vorliegen.

2. Alle Mitglieder erhalten nächstens mit persönlichem Schreiben ihre EAN-Nummer sowie ein Formular, auf dem sie ihren Beitritt zum Vertrag bestätigen oder verweigern können (Art. 3, Abs. 1 TARMED-Rahmenvertrag, Art. 5, Abs. 1 TARMED-Tarifvertrag). Diese Antwort muss bis zum Verschicken der ersten Rechnung gemäss neuem Tarif, mithin nicht schon am 1. Mai 2003 vorliegen. Wir gehen davon aus, dass immer noch die grösste Zahl unserer Mitglieder beitreten werden. Die positive Urabstimmung genügt aber nicht, wie gelegentlich geäussert, einen Beitritt aller Mitglieder ohne formelle Einzelbestätigung anzunehmen.

3. Der Beitritt wird für das Mitglied dann wirksam, wenn seine Antworten zur Dignitätserhebung vorliegen, also spätestens am 18. Mai 2003. Dies bedeutet nicht, dass die Dignitätserklärung zu diesem Zeitpunkt schon in allen Teilen bereinigt bzw. validiert sein muss.
4. Selbstverständlich ist es möglich, mit jedem der einzelnen Sozialversicherer einen Einzelvertrag abzuschliessen. Dieser wird aber immer auf der TARMED-Struktur beruhen. Besitzstandsgarantie werden die Versicherer nicht gewähren; jeder Einzelvertragnehmer wird den für ihn massgeblichen Taxpunktwert selber aushandeln müssen. Die MTK hat in ihrer Sitzung vom 8. April 2003 diese Positionen ausdrücklich bestätigt; sie betrachtet den Taxpunktwert Fr. 1.- wie auch die Besitzstandsgarantie als Ergebnis der Vertragsverhandlungen mit der FMH und mithin nur in diesem Rahmen bzw. dem entsprechenden Vertrag anwendbar. Abschliessend ist festzuhalten, dass die Einzelvertragsnehmer eine Dignitätsabklärung mindestens analog der aktuell für alle FMH-Mitglieder laufenden Dignitätserhebung absolvieren müssen.

5. Die MTK lehnt jegliche Verhandlungen mit einzelnen Fachgesellschaften ab und betrachtet das Verlangen nach fachbereichsspezifischen Tarifen bzw. Tarifstrukturen als ebenso illusorisch wie irrelevant. 


\title{
Introduction du TARMED dans le domaine LAA/LAM/LAI
}

\author{
Dr H. H. Brunner, président de la FMH
}

Nous vous informons comme suit au sujet de l'introduction du nouveau tarif AA/AM/AI:

1. Les formulaires de facturation à l'intention de tous les assureurs sociaux fédéraux sont sous toit et en phase de production. Ces formulaires seront à disposition dans les délais.

2. Tous nos membres vont recevoir sous peu une lettre personnelle avec leur code EAN ainsi qu'un formulaire, sur lequel ils pourront confirmer leur adhésion à la Convention (art. 3, al. 1 de la convention-cadre TARMED; art. 5, al. 1 de la convention tarifaire TARMED). Cette réponse doit être en notre possession d'ici à l'envoi de la première facture selon le nouveau tarif, et donc à une date qui peut être ultérieure au $1^{\mathrm{er}}$ mai 2003. Nous continuons de partir du principe que la grande majorité de nos membres adhérera à la Convention. Contrairement à ce qui a parfois été rapporté, la votation générale ne suffit pas à entériner l'adhésion de tous nos membres sans confirmation individuelle et formelle.

3 L'adhésion d'un membre à la convention prend effet au moment de la mise en vigueur des conventions-cadre TARMED (LAMal ou LAA / LAM / LAI), et son autorisation de facturer devient effective dès l'arrivée de ses réponses au recensement de la valeur intrinsèque, donc au plus tard le 18 mai 2003. Cela ne signifie pas que le recensement de la valeur intrinsèque doive, à ce moment déjà, être définitif et validé à tout point de vue.
4. Il est bien sûr possible de conclure une convention individuelle avec chacun des assureurs sociaux. Mais celle-ci reposera toujours sur la structure tarifaire TARMED. Les assureurs n'accorderont pas la garantie des droits acquis. Chaque médecin souhaitant un contrat individuel devra négocier luimême la valeur du point applicable dans son cas. Lors de sa séance du 9 avril 2003, la CTM a formellement confirmé ses positions. Elle considère la valeur du point de Fr. 1.- et la garantie des droits acquis comme le résultat des négociations contractuelles avec la FMH et, en conséquence, comme applicable seulement dans ce cadre ou dans celui de la convention concernée. Retenons pour conclure que les médecins désireux de conclure une convention individuelle doivent participer à un recensement de la valeur intrinsèque pour le moins analogue à celui qui prévaut actuellement pour les membres de la FMH.

5. La CTM se refuse à toute négociation individuelle avec des sociétés de discipline médicale. Elle considère qu'exiger un tarif ou une structure tarifaire spécifique aux différentes disciplines est une illusion, et que cette démarche ne revêt aucun sens. 